\title{
Referenciales en la intención y decisión de compra de Smartphone en los estudiantes universitarios de Guayaquil
}

\section{Influence of preferential common factors on intention and decision to purchase smartphones in University students of Guayaquil}

Nicolás Armando Sumba Nacipucha

Nicole Carolina Rodríguez Andrade

Universidad Politécnica Salesiana, Ecuador

Autor para correspondencia: nsumba@ups.edu.ec; nrodrigueza@est.ups.edu.ec Fecha de recepción: 15 de diciembre 2017 - Fecha de aceptación: 26 de febrero de 2018

\section{Resumen}

La vorágine de modelos y marcas en el mercado de teléfonos inteligentes obliga al consumidor a enfrentar el proceso de decisión de compra ante un abanico de dispositivos con variedad de prestaciones y precios, esta investigación examina la intención y decisión de compra de smartphones, así como los factores exógenos y endógenos que influyen en los estudiantes universitarios guayaquileños cuando afrontan estos procesos. Se realiza la aplicación de cuestionario a una muestra en seis diferentes centros universitarios, así también se empleó la observación científica afrontada como proceso objetivo y sistémico. Uno de los resultados identifica la necesidad de los estudiantes universitarios de disponer de un smartphone como herramienta tecnológica que aparte de brindarle una serie de prestaciones, le confiere una percepción de aceptación y estatus social, el trabajo expone los resultados a fin de que puedan ser usados como referencia para futuras estrategias de marketing por parte de las marcas.

Palabras clave: comportamiento del consumidor; decisión de compra; motivación; status social y comunicación

\begin{abstract}
The whirlwind of models and brands in the smartphone market forces the consumer to face the decision of purchase process before a range of devices with a variety of benefits and prices, this research examines the intention and decision to purchase smartphones, as well as the exogenous and endogenous factors that influence university students in Guayaquil when they face these processes. The questionnaire was applied to a sample in six different university centers, and scientific observation was also used as an objective and systemic process. One of the results identifies the need of university students to have a smartphone as a technological tool that, apart from providing a series of benefits, confers a perception of acceptance and social status, the work exposes the results so that they can be used as a reference for future marketing strategies by brands.
\end{abstract}

Key words: behavior of consumers; a decision of purchase; motivation; social status and communication 


\section{Introducción}

El comportamiento del consumidor es una de las disciplinas del marketing sobre la cual las empresas han venido trabajando durante las últimas décadas con la finalidad de desarrollar estrategias empresariales que involucren productos o servicios que satisfagan los requerimientos y exigencias del mercado. Esto cobra sentido, dado que los consumidores son quienes determinan las ventas y los beneficios de las empresas con sus decisiones de compra (Descals, 2012).

El mercado de los smartphones es uno de los de mayor expansión en el Ecuador durante los últimos años, en el gráfico 1 se evidencia el crecimiento pronunciado de posesión de dispositivos smartphone a nivel nacional. En el año 2016 se registró un incremento de 15,2\% en la cantidad de personas que disponen de un teléfono inteligente, al pasar del 37,7\% en el año 2015 al 52,9\% en el año 2016. (INEC, 2016).

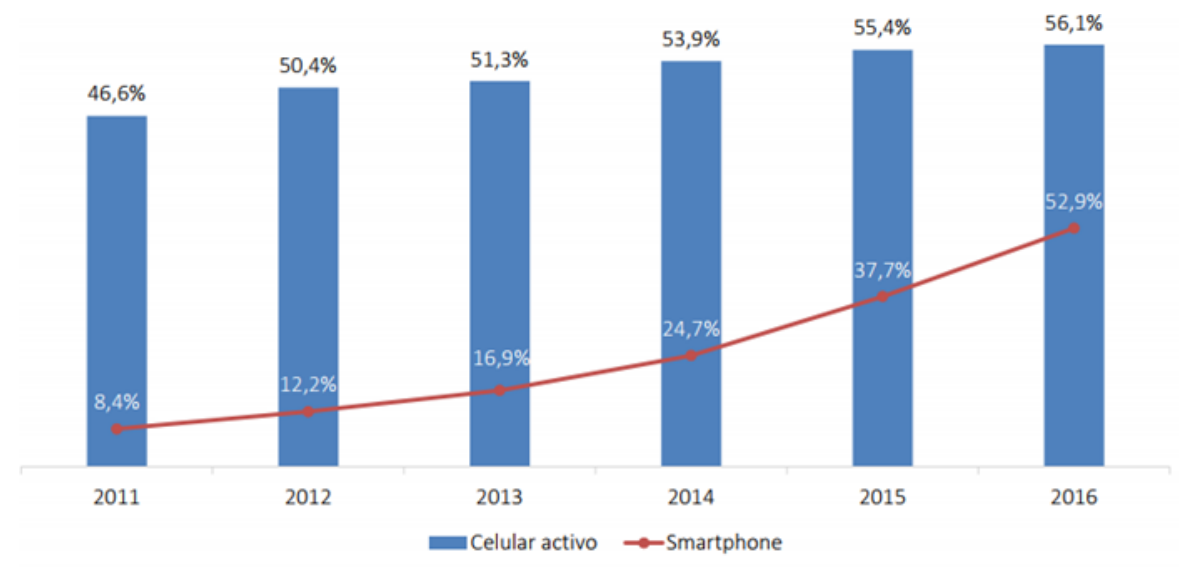

Figura 1. Porcentaje de personas que tienen teléfono inteligente (smartphone) en el Ecuador. Fuente: Encuesta Nacional de Empleo Desempleo y Subempleo - ENEMDU (2014-2016)

Uno de los segmentos de mercado que ha aportado a este crecimiento sostenido son los jóvenes universitarios, esto se puede comprobar al recorrer algún centro de educación superior y observar a estudiantes empleando su smartphone para realizar alguna actividad académica, de comunicación o entretenimiento.

En este sentido, el presente artículo tiene como objetivo, identificar los factores internos y externos que influyen en la decisión de compra de smartphones en los estudiantes universitarios de la ciudad de Guayaquil y diagnosticar la actitud de comportamiento de compra que tiene el consumidor en Guayaquil.

\section{Comportamiento del Consumidor}

La American Marketing Asociation (2014) define la conducta del consumidor como el comportamiento del consumidor o tomador de decisiones en el mercado de productos bienesservicios donde su objetivo básico es lograr el consumo-uso de los productos o servicios que la empresa ofrece al mercado. De acuerdo con lo que señala Esteban (2011) “el individuo encuadra 
en una sociedad de empleos con recursos limitados para satisfacer necesidades", considerando que las conductas van relacionadas con la obtención, uso y consumo de bienes o servicios, trata de comprender, explicar, predecir acciones humanas relacionadas con el consumo.

\section{Factores del comportamiento de decisión de compra}

\section{Factores Externos}

Son denominados así porque influyen en el comportamiento de una persona, como su rutina diaria y entorno; además se encuentran ligados a estos factores como la familia, cultura, subcultura, socioeconómico, ocupación, lo cuales conllevan a una decisión quizás aceptada en los diferentes entornos que los rodean (Yépez, 2016).

La familia es la organización de consumo más importante de la sociedad porque la influencia de padre, madre y niños o adolescentes compromete la adquisición de un producto para su necesidad diaria (Kotler, 2011). En este sentido, se analiza que la influencia de la familia en el comportamiento del consumidor se enfatiza por la tendencia y la frecuencia de consumo de determinados productos en el hogar, así como el proceso de socialización con los miembros del núcleo familiar al respecto de los productos de consumo.

Por otra parte, también constan la cultura y la subcultura, cada cultura consta de subculturas, es decir son más pequeñas y proporcionan a sus miembros una identificación y socialización con la sociedad. Muchas marcas en la actualidad se dirigen a subculturas específicas como consumidores con diferentes creencias religiosas, grupos de referencia, estatus, clase social adaptados a sus necesidades y preferencias específicas. Los factores externos incluyen agentes como; la familia, los grupos de convivencias, grupos de referencia, las clases sociales, la cultura, subcultura, valor agregado, competencia y renovación.

\section{Factores internos en el comportamiento del consumidor}

Hasta ahora se han revisado los estímulos del entorno externo, sin embargo no son los únicos que intervienen en el proceso de compra. El cliente también se ve influenciado por los factores internos, los cuales pueden ser clasificados por grupos. El primero de ellos son los aspectos personales que consisten en los elementos demográficos como: edad, sexo, ciclo de vida, situación económica, ocupación, estilo de vida, personalidad y auto concepto. El segundo grupo corresponde a los aspectos psicológicos, es lo que el consumidor percibe del producto o servicio adquiridos, o también las decisiones de compra de las personas. En los factores psicológicos se incluye la motivación, percepción, aprendizaje, creencias y actitudes. El estudio de la psicología en el proceso de consumo se refiere a la forma como compran los individuos, grupos u organizaciones que usan para seleccionar, comprar, usar y desechar los productos o servicios, ideas o experiencias para satisfacer sus necesidades, además el impacto que tiene el consumidor y la sociedad (Galindo, 2010). La motivación, se refiere a la conducta del miembro en la sociedad de la información que tiene cuatro dimensiones: el consumo, la comunicación, la producción y la navegación (Blokhin, 2013).

\section{Decisión de Compra}


Foscht y Swoboda (2011) indican que cada consumidor debe tomar una decisión cuando piensa en adquirir un producto o servicio. Prácticamente, la decisión de compra va cogida de la mano con el comportamiento, de tal manera que las teorías y modelos sobre la decisión de compra aportan la investigación en el área del comportamiento del consumidor

\section{Etapas de la Decisión de Compra}

Un error común para los individuos ajenos a la mercadotecnia es conceptuar que el proceso de compra se delimita en la adquisición de un producto (Montalvo, 2015).

Kollat y Blackwell (1973) fueron los primeros autores que estudiaron los modelos procesoriales los cuales analizan las fases de decisión de compra y comportamiento del consumidor. Sus cinco modelos giraron en torno a las compras que impulsan y pudieran generase automáticamente en el comprador, motivo por el cual desde su publicación y hasta la actualidad son utilizadas aunque existe una variación por fases o etapas del proceso de compra.

Posteriormente, el modelo titulado EBM Engel, Blackwell y Miniard (2001) se basó en los cincos modelos de KB que involucra el reconocimiento del problema, búsqueda de información, evaluación alternativa, elección y conducta posterior a la compra. Después de diversos cambios en los modelos de decisión de compra hoy en día su uso ha cambiado de fases a etapas.

En las etapas de la decisión de compra el conjunto de actuaciones permite al individuo encontrar la solución al problema. Estas etapas son: Reconocimiento del problema, Búsqueda de la información, Evaluación de Alternativas, Decisión de Compra, Conducta post-compra.

Por lo que respecta a la satisfacción que experimenta un usuario al adquirir un producto o servicio, se ha definido en la literatura desde diferentes planteamientos utilizándose desde enfoques cognitivos y/o afectivos (Oliver ,1997). Es tanto así, que desde un enfoque afectivo la satisfacción se define como el cumplimiento placentero de una necesidad, deseo o meta. De esta forma la satisfacción concibe como una respuesta global del consumidor a la experiencia completa a un momento posterior a la compra. Por otra parte, la insatisfacción del consumidor se ha estudiado profundamente desde la percepción del consumidor. Visto desde esta perspectiva, el concepto de insatisfacción se asemeja al de la satisfacción, dado que es una respuesta que puede ser afectiva, cognitiva e incluso comportamental que se enfoca en un aspecto particular de la experiencia de la compra o del consumo, y que se da en un momento determinado; solo con un carácter opuesto a la satisfacción. (Mora, 2011)

\section{Métodos}

El estudio realizado es de carácter exploratorio, dado que intenta identificar los diferentes factores que influyen en la decisión de compra de smartphones en los estudiantes universitarios. El enfoque de la investigación es cuantitativo-cualitativo; para la parte cuantitativa se utilizó la encuesta como el instrumento de recolección de datos, y para la parte cualitativa se empleó el método de observación científica de forma sistemática, válida y confiable para describir el comportamiento de los consumidores en el proceso de decisión de compra. 
La población del estudio son los estudiantes universitarios de la ciudad de Guayaquil, que según el INEC fueron 63,333 en el año 2016. Para la obtención de la muestra se empleó la fórmula de tamaño muestral para poblaciones finitas, obteniendo como resultado que se debía aplicar el cuestionario a 202 hombres y 202 mujeres con un total de 404 estudiantes universitarios. Se utilizaron muestreos probabilísticos, tales como el muestreo por conglomerados, en donde cada conglomerado era representado por una universidad radicada en la ciudad de Guayaquil, y dentro de cada conglomerado se aplicó muestreo aleatorio simple de tal forma que de cada conglomerado se obtuviera una cantidad de encuestados proporcional al tamaño total de la muestra.

\section{Resultados}

Al referirse a marcas de smartphones que poseen los estudiantes universitarios, el $97 \%$ de los encuestados señalan a Samsung como la primera marca de Smartphone que recuerdan, es decir esta marca se encuentra en el nivel más alto del top of mind de los consumidores universitarios de la ciudad de Guayaquil. Sin embargo, en el gráfico 2 se puede observar la preferencia de marcas de smartphones de los estudiantes universitarios, en el cual es importante acotar que aun cuando Samsung es la marca líder de teléfonos inteligentes en el mercado guayaquileño, un $16 \%$ de encuestados que actualmente poseen un smartphone de esta marca indicó que en su próxima compra adquirirá un teléfono inteligente de otra marca.

Por otra parte, la marca que mejor expectativa tiene en el sector universitario es Apple, dado que es la marca que tiene una mayor proyección de futura compra por parte de los universitarios.

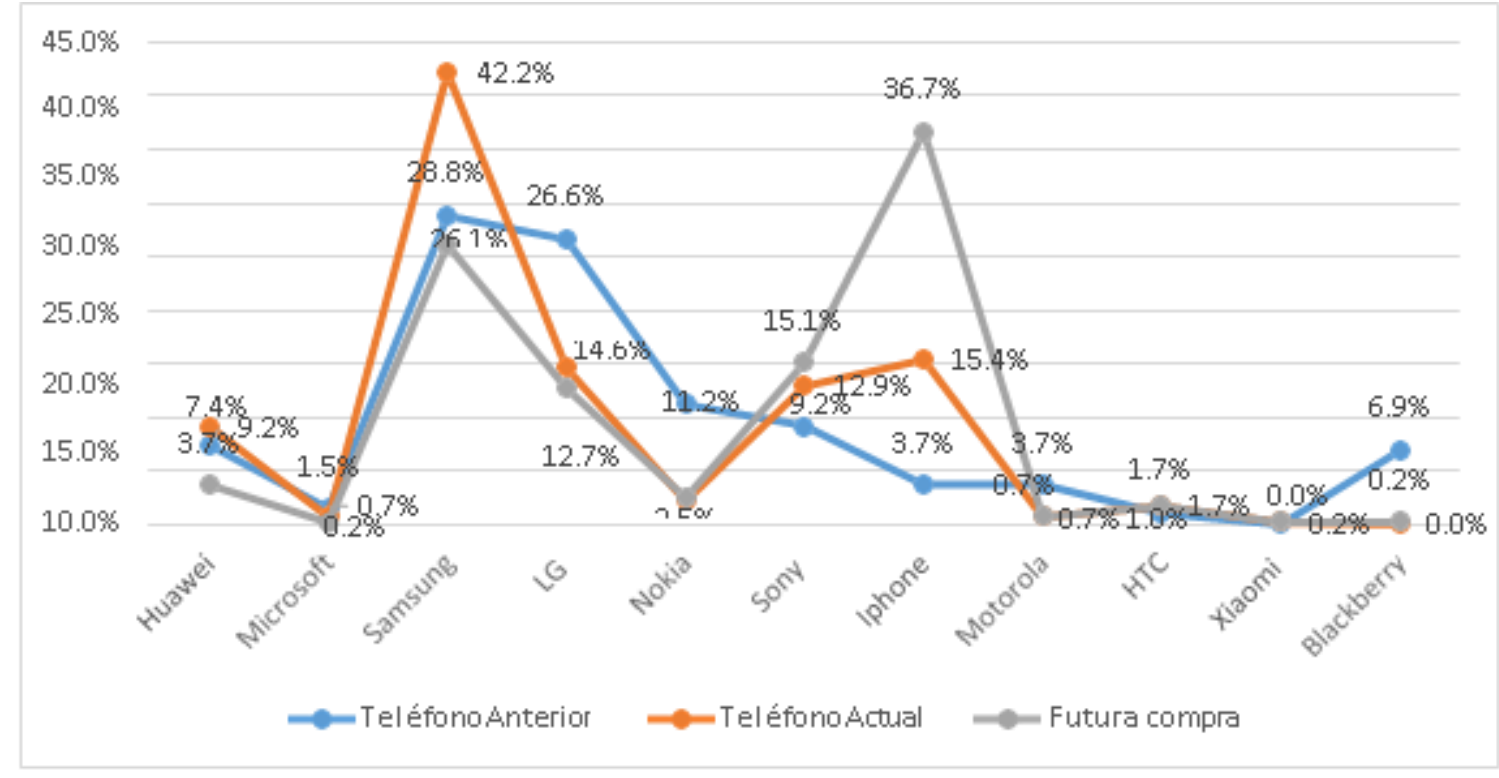

Gráfico 2. Preferencia de marcas de smartphones de consumidores universitarios de la ciudad de Guayaquil

Por otra parte, en el gráfico 3 se observa que la mayoría de los encuestados representados con un $61 \%$ señalan que adquieren un Smartphone a fin de disponer de una herramienta 
tecnológica para el desarrollo de sus actividades, en la actualidad un estudiante universitario desarrolla una serie de actividades académicas desde su dispositivo: revisión de material de estudio, investigaciones en la web, lecciones y deberes en ambientes virtuales.

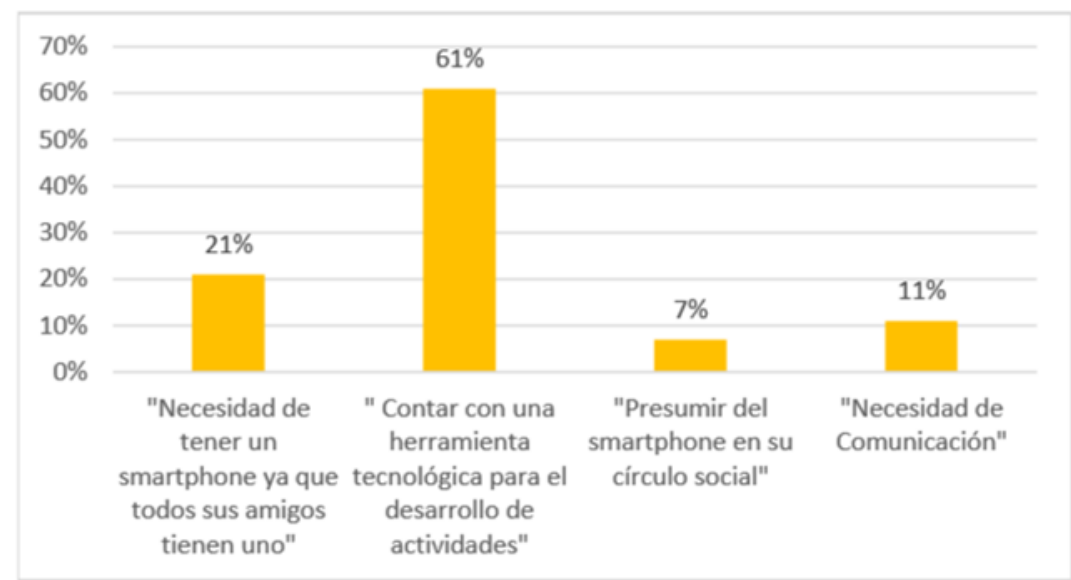

Gráfica 3. Motivos de los estudiantes universitarios para adquirir un smartphone

\section{Conclusiones}

Las marcas de teléfonos inteligentes deben enfocar sus esfuerzos en el desarrollo de productos que satisfagan las necesidades de los diversos tipos de consumidores, y esto generalmente se podrá alcanzar mediante procesos de investigación, desarrollo e innovación que abarquen a toda la empresa.

Es relevante destacar que la mayor parte de estudiantes universitarios se deciden a comprar un teléfono inteligente a fin de disponer de una herramienta tecnológica que les permita desarrollar efectivamente sus actividades laborales, de comunicación, entretenimiento y por supuesto, académicas, y en este último aspecto contribuyen las comunidades universitarias al encontrase en proceso de afianzar el uso de dispositivos tecnológicos dentro del aula de clase. (Cueva, Sumba y López, 2018)

Se puede constatar la presencia de varios factores, tanto exógenos o endógenos al estudiante universitario guayaquileño que influyen en su decisión de compra respecto de smartphones, entre los factores externos a destacar se encuentran: los grupos de referencia, sean estos grupos familiares o de amistades que de una u otra forma ejercen cierta presión en el comportamiento y creencias del individuo, esto se ve reflejado en un importante porcentaje de encuestados que señalan que su motivación más importante para adquirir un Smartphone es la posibilidad de presumirlo en su círculo social, así como contar con uno de estos dispositivos por la sencilla razón de que sus compañeros disponen de smartphones. Es relevante que las marcas de smartphones presten atención a estos factores que influyen en el proceso de decisión de compra y desarrollen estrategias de marketing que permitan satisfacer los requerimientos de los consumidores en este mercado tan dinámico.

\section{Bibliografía}


American Marketing Asociation (2014). [Versión Electrónica] 26 de septiembre del 2014, https://www.ama.org/resources/Pages/Dictionary.aspx?dLetter=P

Blokhin. I, (2013) "Functions and types of behavior of the individual un a network environment", Media. Information. Communication, 1-9.

Cueva Estrada, J., Sumba Nacipucha, N. y López Chila, R. (2018). El uso de los códigos QR: una herramienta alternativa en la tecnología educacional. Revista Publicando, [S.1.], v. 5, n. 14, p. 83-106, mar. 2018. ISSN 1390-9304. Disponible en: <https://rmlconsultores.com/revista/index.php/crv/article/view/982>.

Descals, (2012) "Estrategia de Marketing y Comportamiento Del Consumidor" presentado en A. M Descals (Ed.). Comportamiento Del Consumidor (pp. 14-15). Catalunya, España: UOC.

Engel J.F, Blackwell R.D y Miniard P.W, (2001) “Consumer Behaviour: The Dryden Press Series in Marketing”, 9na Edición, Harcourt College Publisher Pennsylvania, 18 de Noviembre del 2014.

Esteban, J.A, (2011) “Comportamiento del Consumidor". En J.A Esteban (Ed), Madrid: Comportamiento del Consumidor Decisiones y Estrategias de Marketing ESIC, pp.32-35.

Foscht. T y Swoboda. B, (2011) Kaufeverhalten: Grundlagen, Perspektiven, Anwendungen, 4 Edición, Gabler, Wiesbaden

Galindo, A. (2010), "Psicología del consumidor mexicano, Segmento", revista del Instituto Tecnológico de México, No 48.

INEC, Instituto Nacional de Estadísticas y Censo. TIC’S Tecnología de la Información y Educación, [Versión Electrónica] (2016):

http://www.ecuadorencifras.gob.ec/documentos/web-

inec/Estadisticas_Sociales/TIC(2016/170125.Presentación_Tics_2016.pdf

Kollat. D, and R.D. Blackwell. (1973) Consumer Behavior, 2nd Edition, New York: Holt, Rinehart, and Winston

Kotler. P. (2011) Contributions to Marketing Theory and Practice, in Naresh K. Malhotra (ed.) Review of Marketing Research: Special Issue - Marketing Legends (Review of Marketing Research, Volume 8) Emerald Group Publishing Limited, 2011, pp.87 - 120

Montalvo. H. (2015) "La Disonancia Cognitiva Pre-decisoria como estrategia de marketing en el proceso de compra" caso smartphone. Ingeniería de grado en Marketing y Publicidad, febrero Pretty. R, naming names: trademark strategy and beyond: Part one-selecting a brand name, 2008, The Journal of Brand. 
Mora, C. (2011). "La Calidad del Servicio y la Satisfacción al cliente". Revista Brasilera de Marketing, Sao Paulo, 2011, v. 10, n, 2 p 146-162. Recuperado de: http://doi.org/10.5585/remark.v10i2.2212

Oliver. (1997). Satisfaction. A Behaviour Perspective on the consumer, presentado en McGrawHill, Singapur.

Yépez Z. (2016) Razones que motivan la compra de los consumidores de zapatos deportivos del sector de Guayaquil de clase media alta desde los 20 a 30 años de edad. Carrera Administración de Empresas. Universidad Politécnica Salesiana, Guayaquil. 